\title{
Dinámica mitocondrial en las enfermedades neurodegenerativas
}

\author{
Adriana Alarcón-Aguilar, ${ }^{1}$ Paola Maycotte-González, ${ }^{2}$ Paulina Cortés-Hernández, ${ }^{2}$ \\ Norma Edith López-Diazguerrero ${ }^{1}$ y Mina Königsberg ${ }^{1}$ \\ ${ }^{1}$ Universidad Autónoma Metropolitana, Departamento de Ciencias de la Salud, Ciudad de México, México; ${ }^{2}$ Instituto Mexicano del Seguro Social, \\ Centro de Investigación Biomédica de Oriente, Puebla, México
}

\section{Resumen}

Las enfermedades neurodegenerativas son un grupo heterogéneo caracterizado por la disminución gradual, progresiva y selectiva de las funciones del sistema nervioso. La etiología de estas patologías aún se desconoce, sin embargo, se ha propuesto que la función mitocondrial pudiese estar participando en el establecimiento de estas enfermedades, debido al alto requerimiento energético que tienen las neuronas para realizar sus funciones fisiológicas. La mitocondria es un organelo dinámico que puede cambiar su morfología y función en respuesta a diferentes estímulos fisiológicos, por ello se ha empezado a estudiar a la dinámica mitocondrial como uno de los principales reguladores de la supervivencia celular. Este evento comprende diferentes procesos como la generación de nuevas mitocondrias y su eliminación cuando ya no son funcionales, así como los procesos de fusión y fisión mitocondrial y el tráfico de estos organelos en el entorno celular. Todos estos procesos son altamente regulados y tienen como finalidad la óptima funcionalidad de la mitocondria y la homeostasis celular.

PALABRAS CLAVE: Mitocondria. Enfermedades neurodegenerativas. Estrés oxidante. Neuronas.

\begin{abstract}
Neurodegenerative diseases are a group of heterogeneous diseases characterized by a gradual, progressive and selective decrease in nervous system functions. The etiology of these pathologies remains unknown; however, mitochondrial function has been proposed as a common factor that could be involved in the establishment of these diseases, owing to the high energy requirement neurons have in order to carry out their physiological functions. Mitochondria are extremely dynamic organelles that can change their morphology and function in response to different physiological stimuli and, for this reason, mitochondrial dynamics have started being studied as one of cell survival main regulators. This event comprises different processes, such as the generation of new mitochondria and their elimination when they are no longer functional, as well as mitochondrial fusion and fission processes and the traffic of these organelles within the cellular environment. All these processes are highly regulated, and their main purpose is optimal functionality of mitochondria and cellular homeostasis.
\end{abstract}

KEY WORDS: Mitochondria. Neurodegenerative diseases. Oxidative stress. Neurons.

Correspondencia:

Mina Königsberg

E-mail: mkf@xanum.uam.mx
Fecha de recepción: 15-04-2018

Fecha de aceptación: 28-08-2018

DOI: 10.24875/GMM.18004337
Gac Med Mex. 2019;155:276-283

Disponible en PubMed www.gacetamedicademexico.com 


\section{Introducción}

Las enfermedades neurodegenerativas (ENDG) constituyen un grupo heterogéneo caracterizado por la disminución progresiva de las funciones fisiológicas y anatómicas del sistema nervioso. Las ENDG más comunes asociadas con el envejecimiento son las enfermedades de Alzheimer (EA), Parkinson (EP) y Huntington (EH). La etiología de estas patologías aún se desconoce, pero se ha propuesto a la función mitocondrial como un factor común entre ellas debido al alto requerimiento energético que tienen las neuronas para realizar sus funciones fisiológicas. ${ }^{1,2}$ Además, la mitocondria también participa en la señalización intracelular, la producción de especies reactivas de oxígeno (ERO) y la apoptosis. ${ }^{3,4}$

La mitocondria es un organelo altamente dinámico que cambia su morfología y función en respuesta a diferentes estímulos fisiológicos, por lo que la dinámica mitocondrial regula la supervivencia celular. ${ }^{5,6} \mathrm{La}$ dinámica mitocondrial comprende diferentes procesos como la generación de nuevas mitocondrias (biogénesis mitocondrial), la eliminación de estas (mitofagia) y, particularmente, la fusión, fisión, transporte y distribución en la célula (tráfico).

\section{Fisión y fusión mitocondrial}

Los eventos de la dinámica mitocondrial más estudiados son la fusión y la fisión (división) mitocondrial. Estos procesos suceden continuamente y permiten que porciones funcionales de la red mitocondrial se separen y se reubiquen dentro de la célula para la producción de energía. ${ }^{7,8}$ Son esenciales para el mantenimiento y distribución del ADN mitocondrial, el control de calidad del organelo y la regulación de la apoptosis. ${ }^{8,9}$

Las proteínas centrales que ejecutan la fusión y fisión son unas GTPasas altamente conservadas relacionadas con la familia de las dinaminas denominadas DRP (dynamin related proteins). ${ }^{7}$

La proteína 1 relacionada con la dinamina (Drp1) ejecuta la fisión mitocondrial. Drp1 es una proteína citosólica que se recluta y ancla en la membrana externa mitocondrial (MEM) gracias a la presencia de varias proteínas transmembranales como la proteína de fisión 1 (Fis 1). ${ }^{10}$ Drp1 se ensambla para formar una espiral compuesta por cientos de monómeros de Drp1 que se enrollan y envuelven las porciones delgadas de los túbulos mitocondriales, estrangulándolos y escindiendo simultáneamente a las membranas externa e interna. ${ }^{10}$

Durante la fusión también participan GTPasas de la familia de las DRP, a diferencia de la mayoría de los eventos de fusión intracelular que ocupan proteínas tipo SNARE. ${ }^{11}$ La fusión mitocondrial ocurre en dos pasos: primero, las MEM de dos mitocondrias se fusionan a través de la interacción de las mitofusinas (Mfn1/Mfn2) que se encuentran ancladas en estas membranas; en una segunda etapa, la proteína relacionada con la atrofia óptica 1 (Opa1) fusiona las membranas internas mitocondriales (MIM). ${ }^{12,13}$ Estos eventos se pueden separar debido a sus diferentes requisitos energéticos: la fusión de la MIM demanda concentraciones de GTP más elevadas que la fusión de la MEM, pero ambas ocurren solo entre las mitocondrias que mantienen un potencial de membrana (Figura 1, vías color rojo y verde). Las DRP que participan en estos procesos son reguladas por modificaciones postraduccionales. ${ }^{14-21}$

\section{Biogénesis mitocondrial}

La masa mitocondrial puede aumentar en respuesta a diversos estímulos como el ejercicio, las hormonas, la diferenciación y división celular, el estrés oxidante, etcétera. ${ }^{22}$ Debido a su origen endosimbiótico, la generación de mitocondrias requiere la expresión concertada de dos genomas: el nuclear, que aporta más de 1000 proteínas, y el mitocondrial que en los seres humanos codifica solo para 13 proteínas, todas subunidades hidrofóbicas de la cadena respiratoria (más 22 tARN y dos rARN). ${ }^{22,23}$ Dado que muchas proteínas mitocondriales se expresan a partir del genoma nuclear, el proceso de biogénesis depende en gran medida de la importación y el ensamblaje de proteínas en los compartimentos mitocondriales ${ }^{24}(\mathrm{Fi}$ gura 1, vía color azul).

La expresión de los dos genomas está coordinada por el coactivador transcripcional PGC-1 $\alpha$ (coactivador del receptor activado por el proliferador de peroxisoma $1 \alpha$ ), considerado el principal regulador de la biogénesis mitocondrial. ${ }^{25,26}$ PGC- $1 \alpha$ carece de dominios de unión al ADN, por lo que funciona a través de la activación de múltiples factores de transcripción, entre los que destacan factores respiratorios nucleares, receptores activados por proliferadores de peroxisomas y receptores relacionados con estrógenos, que controlan los genes de la fosforilación oxidativa, la oxidación de ácidos grasos y la defensa antioxidante, etcétera. ${ }^{23}$ 


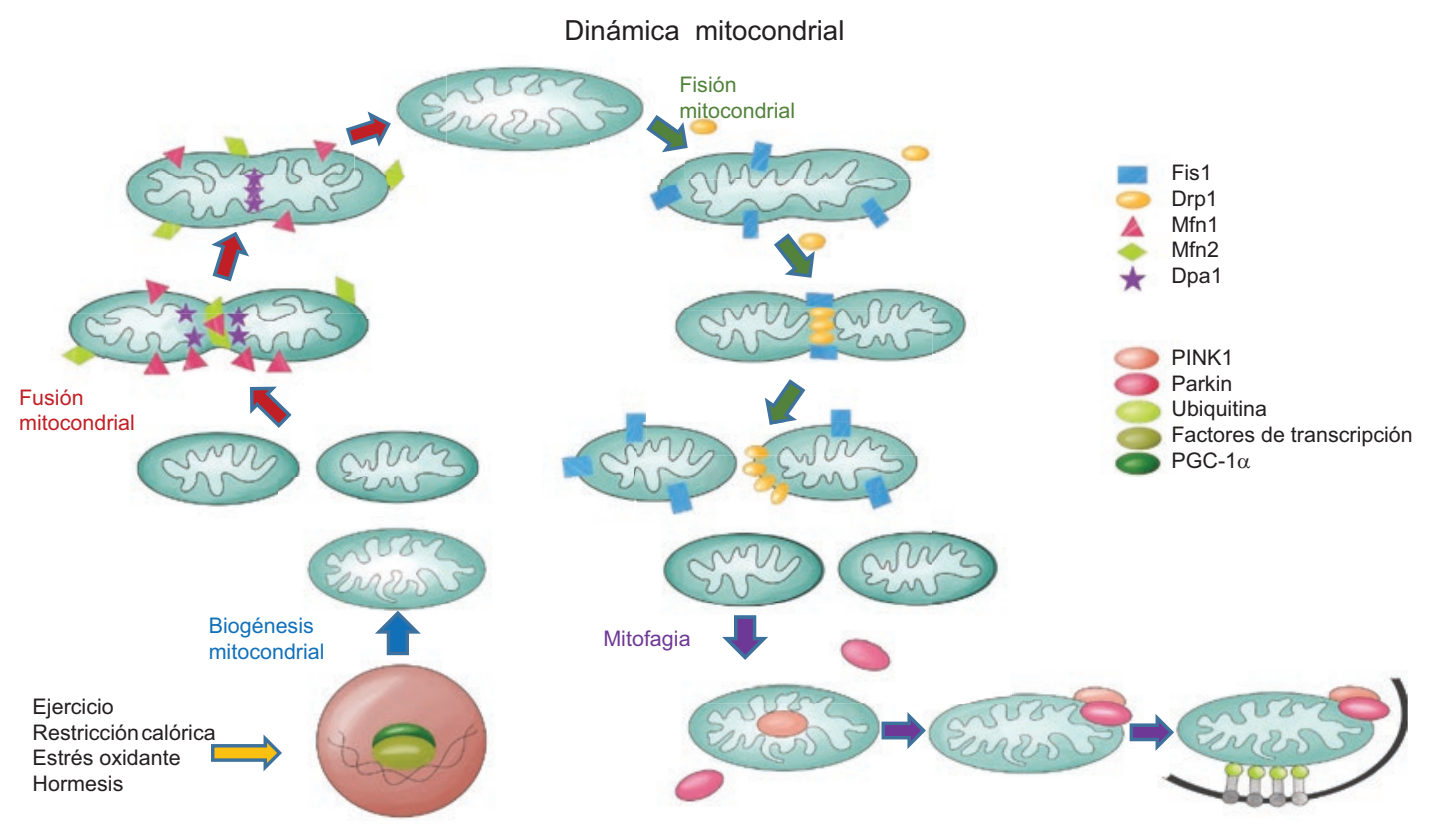

Figura 1. Representación de la dinámica mitocondrial y las proteínas participantes. La vía de fusión se ejemplifica con rojo, la fisión con el color verde, mientras que la biogénesis con azul y la mitofagia con morado.

\section{Mitofagia}

Las mitocondrias se eliminan mediante un proceso regulado llamado mitofagia, una forma de macroautopagia selectiva. La macroautofagia es el mecanismo de degradación donde una vesícula de doble membrana, el autofagosoma, se forma alrededor de los componentes celulares dañados y luego se fusiona con un lisosoma para la degradación del contenido. Cuando las porciones dañadas de la red mitocondrial no pueden generar potencial de membrana, se fisionan y son eliminadas por mitofagia. ${ }^{27,28}$ El mecanismo de mitofagia más estudiado está mediado por las proteínas PINK1 y Parkin. La cinasa PINK1 (serina/ treonina cinsasa inducida por PTEN) se degrada constantemente en las mitocondrias sanas por la proteasa PARL (proteasa tipo romboide asociada con la presenilina), que se inactiva en las mitocondrias despolarizadas, lo que estabiliza PINK1 en la MEM. PINK recluta y fosforila la ubiquitina-ligasa citosólica E3, Parkin, y a su vez Parkin ubiquitina a varias proteínas en la MEM que sirven como señal para reclutar la maquinaria de autofagia, eliminando así a la mitocondria dañada ${ }^{8,28-30}$ (Figura 1, vía color morado).

\section{Tráfico mitocondrial}

El tráfico mitocondrial se refiere al transporte y distribución de las mitocondrias en las células. En particular en las neuronas, la concentración de mitocondrias es mayor en las regiones presinápticas; las mitocondrias se desplazan largas distancias dentro de la neurona para cubrir sus altos requerimientos energéticos. Esto se realiza gracias al acoplamiento entre las proteínas motoras y las adaptadoras. Las proteínas adaptadoras, mejor caracterizadas que unen las mitocondrias a la cadena pesada de la cinesina son Miro y Milton. Miro es una Rho-GTPasa y en mamíferos tiene dos isoformas: Miro-1 y Miro-2. Igualmente hay dos ortólogos de Milton, TRAK1 y TRAK2. ${ }^{31}$

Estos procesos deben estar regulados para que las células se encuentren en homeostasis. Las alteraciones en la dinámica mitocondrial se han relacionado con la etiología de varias enfermedades neurodegenerativas (Tabla 1).

\section{Enfermedad de Alzheimer}

La EA es una de las neuropatologías más recurrentes en los adultos mayores. Se ha reportado que 60 a $80 \%$ de los casos de demencia están asociados con ella, ${ }^{32}$ que se caracteriza por la pérdida progresiva de neuronas en regiones cerebrales relacionadas con funciones como la memoria, el aprendizaje, la consciencia y el lenguaje. Los marcadores neuropatológicos característicos incluyen los ovillos neurofibrilares, agregados intracelulares compuestos por la forma hiperfosforilada de la proteína Tau asociada 
Tabla 1. Características de las tres principales enfermedades neurodegenerativas

\begin{tabular}{|c|c|c|c|}
\hline Característica & Alzheimer & Parkinson & Huntington \\
\hline Origen & Hereditaria y medioambiental & Hereditaria y medioambiental & Hereditaria \\
\hline $\begin{array}{l}\text { Manifestaciones de la } \\
\text { enfermedad }\end{array}$ & $\begin{array}{l}\text { Pérdida de la memoria, el } \\
\text { aprendizaje, la consciencia y } \\
\text { el lenguaje }\end{array}$ & $\begin{array}{l}\text { Alteraciones motoras como la } \\
\text { bradicinesia y temblor en reposo y } \\
\text { rigidez }\end{array}$ & $\begin{array}{l}\text { Disfunción motora, movimientos } \\
\text { involuntarios, distonía, deterioro cognitivo, } \\
\text { deterioro intelectual y trastornos } \\
\text { emocionales }\end{array}$ \\
\hline $\begin{array}{l}\text { Regiones afectadas del } \\
\text { cerebro }\end{array}$ & Hipocampo & $\begin{array}{l}\text { Degeneración de las neuronas } \\
\text { dopaminérgicas en la sustancia nigra }\end{array}$ & $\begin{array}{l}\text { El caudado y el putamen del cuerpo } \\
\text { estriado, la corteza cerebral, el } \\
\text { hipocampo, el hipotálamo y el subtálamo }\end{array}$ \\
\hline Marcadores & $\begin{array}{l}\text { Formación de ovillos } \\
\text { neurofibrilares y agregados de } \\
\text { fibras del péptido amiloide- } \beta\end{array}$ & $\begin{array}{l}\text { Presencia de inclusiones } \\
\text { intracitoplásmicas (cuerpos de Lewy). } \\
\text { Formación de placas beta amiloides } \\
\text { y ovillos neurofibrilares }\end{array}$ & Presencia de huntingtina mutada ( $\mathrm{mHtt})$ \\
\hline Alteraciones fisiológicas & $\begin{array}{l}\text { - Disfunción mitocondrial } \\
\text { - Estrés oxidante } \\
\text { - Apoptosis } \\
\text { - Transmisión sináptica }\end{array}$ & $\begin{array}{l}\text { - Disfunción mitocondrial } \\
\text { - Estrés oxidante } \\
\text { - Apoptosis } \\
\text { - Autofagia vía lisosoma }\end{array}$ & $\begin{array}{l}\text { - Disfunción mitocondrial } \\
\text { - Estrés oxidante } \\
\text { - Apoptosis }\end{array}$ \\
\hline Dinámica mitocondrial & $\begin{array}{l}\text { - Fisión mitocondrial } \\
\text { - Drp1 } \\
\text { - Fusión mitocondrial } \\
\text { - Mfn2 } \\
\text { - Opa1 }\end{array}$ & $\begin{array}{l}\text { - Fisión mitocondrial } \\
\text { - Drp1 } \\
\text { - Fusión mitocondrial } \\
\text { - Mfn1 y Mfn2 } \\
\text { - Opa1 }\end{array}$ & $\begin{array}{l}\text { - Fisión mitocondrial } \\
\text { - Drp1 } \\
\text { - Fis1 } \\
\text { - Fusión mitocondrial } \\
\text { - Opa1 } \\
\text { - Mfn1 y Mfn2 } \\
\text { - Tom40 }\end{array}$ \\
\hline Biogénesis mitocondrial & $-P G C-1 \alpha$ & $-P G C-1 \alpha$ & $\begin{array}{l}\text { - PGC- } 1 \alpha \\
\text { - TFAM }\end{array}$ \\
\hline Mitofagia & $\begin{array}{l}\text { - Por acumulación intracelular } \\
\text { de Tau }\end{array}$ & $\begin{array}{l}\text { - En general, y con mutaciones en } \\
\text { PINK1 y Parkina en EP hereditaria }\end{array}$ & $\begin{array}{l}\text { - Por falta de interacción de Htt con p62 } \\
\text { para reconocer proteínas que serán } \\
\text { ubiquitinadas }\end{array}$ \\
\hline
\end{tabular}

con los microtúbulos y las placas seniles, que son lesiones extracelulares formadas por agregados de fibras del péptido amiloide- $\beta .^{32,33}$

Recientemente se ha reportado que existen cambios morfológicos en los cerebros de pacientes con $E A$, caracterizados por la presencia de mitocondrias fragmentadas, lo cual se asocia con alta actividad de Drp1. La fisión mitocondrial excesiva contribuye a la disfunción mitocondrial y deficiencia en la transmisión sináptica, lo que lleva a un aumento en la muerte celular neuronal. ${ }^{34,35}$ En un modelo de doble transgénico de la EA (proteína precursora del amiloide 1/ presenilina 1), se observó que al inhibir a Drp1 se impedía la fragmentación mitocondrial, la pérdida del potencial de membrana mitocondrial, la producción de ERO, la reducción de ATP y la función sináptica en neuronas tratadas con el péptido amiloide- $\beta$. Además, la inhibición de Drp1 mejoró significativamente el aprendizaje y la memoria, disminuyó la peroxidación lipídica y la deposición de amiloide- $\beta$. Por ello se propuso a los inhibidores de la fisión mitocondrial (inhibidores de Drp1), como una nueva estrategia terapéutica para la EA. ${ }^{36}$ Lo anterior se confirmó en otro trabajo realizado en el hipocampo de ratones de 12 meses que sobreexpresaban la proteína Tau; se observó incremento en el número de mitocondrias con menor longitud a las normales, niveles incrementados de las proteínas de fisión Drp1 y Fis1 y niveles disminuidos de proteínas de fusión Mfn1, Mfn2 y Opa1. ${ }^{37}$

La biogénesis mitocondrial también está alterada en el cerebro de personas con EA. Diferentes estudios han demostrado la reducción del número de mitocondrias en neuronas del hipocampo en el cerebro de pacientes con EA y en células M17 que sobreexpresan la mutante de la proteína precursora del amiloide (APPswe). Asimismo, los niveles de expresión de PGC-1 $\alpha$, NRF1, NRF2 y TFAM se reducen en el hipocampo de personas con EA y en células APPswe M17, lo que sugiere biogénesis mitocondrial reducida. De hecho, las células APPswe M17 mostraron disminución en la proporción de ADN mitocondrial/ADN nuclear, correlacionada con un contenido reducido de 
ATP. Sin embargo, teniendo en cuenta las funciones pleiotrópicas de PGC-1 $\alpha$, aún no queda claro si tales cambios tienen influencia directa en la disfunción mitocondrial durante la EA. ${ }^{38}$

En cuanto a la mitofagia, se encontró que el cerebro de pacientes con EA y de ratones transgénicos que sobreexpresan la proteína Tau humana (hTau), muestran deficiencias en la mitofagia. La sobreexpresión de hTau, altera el potencial de membrana mitocondrial, mientras que los niveles de PINK1 y Parkin disminuyen. También se detectó deterioro dependiente de la cantidad de Tau presente en la fracción mitocondrial de la MEM junto con su acumulación citoplásmica. Estos datos sugieren que la acumulación intracelular de hTau induce deficiencia en la mitofagia por su inserción directa en la membrana mitocondrial, alterando el potencial de membrana, afectando el cociente PINK1/Parkin. ${ }^{39}$ Otro estudio con ratones 5 XFAD que acumulan a la proteína amiloide- $\beta$ reportó incremento en Parkin y en el reclutamiento de la proteína LC3II en mitocondrias sinaptosomales dependiendo de la edad, así como niveles incrementados de Drp1 y disminuidos de Mfn2 y Opa1, que apuntan a incremento en la mitofagia en las mitocondrias sinaptosomales, lo que explicaría parcialmente la deficiencia en la sinapsis en la EA..$^{40}$

Las proteínas Miro, involucradas en la regulación del transporte mitocondrial, se han asociado con trastornos neurodegenerativos y del neurodesarrollo, como en la EA. ${ }^{41}$ Aunque no se ha demostrado asociación directa entre Miro y EA, se sabe que los niveles de Miro están regulados negativamente en la mutación E280A de presenilina 1, asociada con la EA. ${ }^{42}$ Estudios recientes revelaron que los cerebros de pacientes con EA acumulan mitocondrias defectuosas en las regiones distales de las neuronas, lo que se correlaciona con transporte mitocondrial incorrecto, dificultando que las mitocondrias dañadas puedan ser transportadas al soma para su degradación, generando depuración mitofágica inadecuada, ${ }^{31}$ sin embargo, la relación de los defectos del tráfico mitocondrial y la EA todavía necesita ser investigada más a fondo para establecer posibles tratamientos.

\section{Enfermedad de Parkinson}

La EP es la segunda ENDG más común y se asocia con alteraciones motoras como bradicinesia, temblor en reposo y rigidez. Se caracteriza por degeneración progresiva de las neuronas dopaminérgicas (NDA) en la sustancia negra y la presencia de inclusiones intracitoplásmicas llamadas cuerpos de Lewy. ${ }^{43,44}$

La disfunción mitocondrial desempeña un papel fundamental en la patogénesis de la EP, tanto en las formas esporádicas como en las de origen hereditario y puede ser causada por defectos bioenergéticos, mutaciones en el ADN mitocondrial, mutaciones en el ADN nuclear vinculadas a los genes mitocondriales, cambios en los procesos de fusión o fisión y alteraciones en el tráfico mitocondrial. Una de las razones propuestas para la vulnerabilidad de las NDA de la sustancia negra es que basalmente contienen menor número de mitocondrias en comparación con otras neuronas del mesencéfalo. ${ }^{45}$ Se ha reportado que alteraciones en la dinámica mitocondrial incrementan la producción de ERO y disminuyen el potencial de membrana mitocondrial, lo que podría potenciar la acumulación de mitocondrias disfuncionales. Sin embargo, la fragmentación mitocondrial excesiva también se ha asociado con la patología de la EP esporádica.

En un estudio comparando muestras de pacientes con EP idiopática y animales expuestos al paraquat (un herbicida empleado como modelo de la enfermedad), se observó un incremento en la expresión de Drp1 y una reducción en la expresión de Mfn1/Mfn2 en la sustancia nigra, acompañada de un incremento en la fragmentación y en la disfunción mitocondrial. Al sobreexpresar a Mfn2 se eliminó la fragmentación mitocondrial y se protegió a las neuronas contra la disfunción inducida por paraquat. También se observó que el daño oxidante inducido por paraquat y la pérdida selectiva de NDA en la sustancia nigra y en las terminales axonales en el cuerpo estriado disminuyeron en ratones transgénicos que sobreexpresaban hMfn2. ${ }^{46}$

Se ha demostrado que las alteraciones en la estructura de OPA1 están relacionadas con la fragmentación mitocondrial observada en modelos celulares de la EP esporádicos, lo cual se asocia con aumento en la expresión de Drp1. Para demostrar la relevancia de estas alteraciones se sobreexpresó a OPA1 y se inhibió la expresión de Drp1. En este estudio, la sobreexpresión de OPA1 no modificó la fragmentación mitocondrial ni tampoco disminuyó los niveles de ERO, pero se encontró incremento en la elongación e interconectividad mitocondrial, lo que mantuvo el potencial de membrana. ${ }^{47}$

Por otro lado, los cerebros de pacientes con EP tienen capacidad respiratoria y biogénesis mitocondrial disminuida que podría inducir la formación de placas 
betaamiloides y ovillos neurofibrilares. Asimismo, las ERO generadas por las redes mitocondriales disfuncionales pueden activar las vías de señalización que inducen la muerte celular, mientras que las proteínas agregadas contribuyen a neuroinflamación. ${ }^{48}$

Se han relacionado las mutaciones en la proteína Parkin con la EP autosómica recesiva de inicio temprano. En estudios con ratones adultos C57BL, la disminución de Parkin en la región media ventral del cerebro indujo la pérdida de NDA dependiendo de la edad. Este proceso está sujeto a la acumulación del sustrato de Parkin llamado PARIS, también conocido como la proteína dedos de cinc 746 (ZNF746) y a la represión transcripcional de PGC-1 $\alpha$. En los ratones knockout de Parkin, en el cerebro medio ventral del ratón adulto $\mathrm{C} 57 \mathrm{BL}$ disminuyó el tamaño y numero de mitocondrias, lo que se previno mediante el silenciamiento de PARIS. Por otra parte, la sobreexpresión de PARIS en el mesencéfalo ventral del ratón disminuyó la activación de PGC-1 $\alpha$ y el número de mitocondrias. Estos mismos resultados fueron demostrados en la línea celular SH-SY5Y. Los datos en conjunto sugieren que la pérdida de Parkin afecta la biogénesis mitocondrial, lo que disminuye la función respiratoria debido a menor número de mitocondrias y a muerte celular. ${ }^{49}$

Se sabe que la función mitocondrial y lisosomal disminuyen con la edad, ${ }^{50,51}$ lo que concuerda con que la edad es el factor de riesgo mayor para desarrollar la EP. El deterioro de la mitofagia y de la actividad lisosomal se han asociado con incremento en la expresión de la $\alpha$-sinucleína observado en la EP. ${ }^{51,52}$ Los factores de transcripción NRF2 y TFEB que desempeñan un papel en la biogénesis mitocondrial y lisosomal, respectivamente, pueden regular a p62/ SQSMT1, proteína que marca a las mitocondrias dañadas para su degradación por mitofagia. Se ha reportado que las células con mayor proteína TFEB tienen niveles de expresión más altos del ARNm de PGC-1 $\alpha$. Por lo tanto, se ha propuesto que las estrategias para aumentar el TFEB pueden incrementar el número de mitocondrias y evitar la disfunción mitocondrial en la EP. ${ }^{53}$

\section{Enfermedad de Huntington}

La EH es una patología que afecta a algunas personas en la mitad de su vida. Es de carácter genético, con una herencia autosómica dominante. La EH se caracteriza por disfunción motora, movimientos involuntarios, distonía, deterioro cognitivo y trastornos emocionales. ${ }^{54,55}$ Esta enfermedad tiene una baja prevalencia ya que afecta entre 10 y 13 personas por cada 100000 habitantes. ${ }^{56}$ Las principales áreas del cerebro que se afectan incluyen el caudado y el putamen del cuerpo estriado, la corteza cerebral, el hipocampo, el hipotálamo y el subtálamo. ${ }^{54,57}$ Se ha observado disminución neuronal en estas regiones del cerebro hasta en $80 \%$ de los pacientes con $\mathrm{EH}$ severa. ${ }^{58}$

La EH es causada por expansión de repetición de trinucleótidos de CAG inestable en el exón 1 del gen Huntingtin $(H t t)$ que codifica una forma mutante de la proteína huntingtina $(\mathrm{Htt})$. La presencia de más de 40 repeticiones de CAG se reconoce como la huntingtina mutante ( $\mathrm{mHtt}$ ), que causa la enfermedad. EI inicio de la $\mathrm{EH}$ generalmente ocurre en la mediana edad, seguido de 15 a 20 años de progresión de la enfermedad. ${ }^{59}$

Diferentes líneas de investigación apoyan la hipótesis de que la alteración de la función mitocondrial y energética son importantes en la fisiopatología de la EH. ${ }^{60-62}$ De forma similar a otras ENDG, se demostró que la fisión mitocondrial se encontraba incrementada en muestras de pacientes con diferentes grados de la EH, ya que los niveles de Drp1 se encontraban aumentados y los de Mfn1 disminuidos. Asimismo, se reportó disminución en la expresión de PGC-1 $\alpha$ y TFAM. ${ }^{62}$ Shirendeb et al. confirmaron estos resultados: Fis1 y Drp1 estaban aumentados, mientras que Opa1, Mfn1, Mfn2 y Tom40 se encontraban disminuidas en pacientes con grado III y IV de la EH en comparación con pacientes sin la enfermedad..$^{63}$ En cultivos primarios de neuronas provenientes de ratones BACHD que expresan mHtt, así como en muestras de pacientes con $\mathrm{EH}$ se encontró que el incremento en la fragmentación mitocondrial dependiente de Drp1 se debía a su interacción con la mHtt. También se observó incremento en los niveles de expresión de Drp1 y Fis1, así como una disminución de Mfn1/Mfn2. ${ }^{63}$

Las alteraciones en la dinámica mitocondrial como el desbalance entre la fisión y la fusión pueden ser hasta cierto punto compensadas por incremento en la biogénesis mitocondrial. Sin embargo, se ha reportado que la mHtt disminuye la función mitocondrial inhibiendo la expresión de PGC-1 $\alpha .{ }^{64-66} \mathrm{La} \mathrm{mHtt}$ reprime la transcripción génica de PGC-1 $\alpha$ al unirse con el promotor e interferir con la ruta transcripcional dependiente de CREB/TAF4, la cual participa críticamente en la expresión de los genes regulados por PGC-1 $\alpha$. Se ha encontrado que la sobreexpresión de PGC-1 $\alpha$ invierte parcialmente los efectos tóxicos de 
la mHtt en cultivos primarios de neuronas estriatales, y que la administración de PGC-1 $\alpha$ mediada por lentivirus en el cuerpo estriado proporciona neuroprotección en los ratones transgénicos de la $\mathrm{EH} .{ }^{64}$

Por otra parte, se ha sugerido que la eliminación de mitocondrias defectuosas está comprometida en la EH. La eliminación de mitocondrias defectuosas depende de la actividad mitofágica que involucra a la vía PINK1/Parkin. ${ }^{67-69}$ Recientemente se reportaron fallas en el proceso de mitofagia en moscas que expresaban $\mathrm{mHtt}$, como los modelos de Htt-ex1p-Q93 neuronal y en la línea celular del estriado HdhQ111, los cuales se asocian con disminución de las proteínas ubiquitinadas en las mitocondrias que serán degradadas en los autofagosomas..$^{70}$ El mismo estudio demostró que la sobreexpresión de PINK1 funcionaba como neuroprotector en moscas y restablecía la mitofagia en células HdhQ111. Se ha propuesto que la Htt de tipo silvestre desempeña un papel fisiológico en la mitofagia posiblemente por su interacción con receptores de autofagia. Específicamente, se reportó que el carboxilo terminal de la Htt interactúa con p62 para facilitar el reconocimiento de proteínas que deben ser ubiquitinadas, lo que es relevante para la mitofagia ya que las células deficientes en Htt presentan niveles de mitofagia más bajos. ${ }^{71}$ El transporte mitocondrial a lo largo de los microtúbulos también disminuye en cultivos primarios de neuronas corticales que expresan $\mathrm{mHtt},{ }^{72,73}$ ya que los agregados de $\mathrm{mHtt}$ pueden bloquear el transporte a los axones, secuestrar proteínas motoras e inhibir la formación de los complejos de tráfico mitocondrial. ${ }^{73,74}$

\section{Conclusión}

Los reportes sobre la relación de la dinámica mitocondrial y las ENDG proponen un nuevo campo de investigación para desarrollar tratamientos alternativos que aminoren la sintomatología y el deterioro. Los trabajos aquí descritos muestran interesantes resultados, ya que la sobreexpresión de proteínas vinculadas con la dinámica mitocondrial, en particular las de fusión, es alentadora puesto que se ha observado mejoría en el suministro energético y en el transporte mitocondrial, así como en la disminución en la fragmentación. No obstante, el reto para investigaciones posteriores será desarrollar mecanismos que impidan las interacciones de las proteínas que se expresan aberrantemente en las ENDG con las proteínas que participan en la dinámica mitocondrial y reducir el daño mitocondrial para dar a los pacientes una mejor calidad de vida.

\section{Agradecimientos}

Este trabajo fue apoyado con financiamiento del Conacyt CB-2015-01-255591 para Norma Edith López-Diazguerrero y FON.INST/298/2016 para Mina Königsberg, así como por la Red Temática de Investigación en Salud y Desarrollo Social, del Conacyt.

\section{Referencias}

1. Grimm A, Eckert A. Brain aging and neurodegeneration: from a mitochondrial point of view. J Neurochem. 2017;143:418-431.

2. Shah SZA, Zhao D, Hussain T, Yang L. Role of the AMPK pathway in promoting autophagic flux via modulating mitochondrial dynamics in neurodegenerative diseases: insight into prion diseases. Ageing Res Rev. 2017;40:51-63.

3. Chen Y, Zhang H, Zhou HJ, Ji W, Min W. Mitochondrial redox signaling and tumor progression. Cancers (Basel). 2016;8:40.

4. Frohman MA. Role of mitochondrial lipids in guiding fission and fusion. J Mol Med (Berl). 2015;93:263-269.

5. Gao J, Wang L, Liu J, Xie F, Su B, Wang X. Abnormalities of mitochondrial dynamics in neurodegenerative diseases. Antioxidants (Basel). 2017;6:25.

6. Golpich M, Amini E, Mohamed Z, Azman Ali R, Mohamed Ibrahim N, Ahmadiani A. Mitochondrial dysfunction and biogenesis in neurodegenerative diseases: pathogenesis and treatment. CNS Neurosci Ther. 2017;23:5-22.

7. Labbé K, Murley A, Nunnari J. Determinants and functions of mitochondrial behavior. Annu Rev Cell Dev Biol. 2014;30:357-391.

8. Wai T, Langer T. Mitochondrial dynamics and metabolic regulation. Trends Endocrinol Metab. 2016;27:105-117.

9. Youle RJ, van Der-Bliek AM. Mitochondrial fission, fusion, and stress. Science. 2012;337:1062-1065.

10. Lackner LL, Nunnari JM. The molecular mechanism and cellular functions of mitochondrial division. Biochim Biophys Acta. 2009;1792:1138-1144.

11. Jahn R, Scheller RH. SNAREs engines for membrane fusion. Nat Rev Mol Cell Biol. 2006;7:631-643.

12. Meeusen SL, Nunnari J. How mitochondria fuse. Curr Opin Cell Biol. 2005;17:389-394.

13. Hoppins S, Nunnari J. The molecular mechanism of mitochondrial fusion. Biochim Biophys Acta. 2009;1793:20-26.

14. Pernas L, Scorrano L. Mito-morphosis: mitochondrial fusion, fission, and cristae remodeling as key mediators of cellular function. Annu Rev Physiol. 2016;78:505-531.

15. López-Lluch G. Mitochondrial activity and dynamics changes regarding metabolism in ageing and obesity. Mech Ageing Dev. 2017;162:108-121.

16. Horbay R, Bilyy R. Mitochondrial dynamics during cell cycling. Apoptosis. 2016;21:1327-1335.

17. Chan DC. Mitochondria: dynamic organelles in disease, aging, and development. Cell. 2006;125:1241-1252.

18. Campello S, Scorrano L. Mitochondrial shape changes: Orchestrating cell pathophysiology. EMBO Rep. 2010;11:678-684.

19. Friedman JR, Nunnari J. Mitochondrial form and function. Nature. 2014;16;505:335-343.

20. Cho DH, Nakamura T, Lipton SA. Mitochondrial dynamics in cell death and neurodegeneration. Cell Mol Life Sci. 2010;67:3435-3447.

21. Hoppins S. The regulation of mitochondrial dynamics. Curr Opin Cell Biol. 2014;29:46-52.

22. Ventura-Clapier R, Garnier A, Veksler V. Transcriptional control of mitochondrial biogenesis: the central role of PGC-1alpha. Cardiovasc Res. 2008;79:208-217.

23. Scarpulla RC. Nucleus-encoded regulators of mitochondrial function: integration of respiratory chain expression, nutrient sensing and metabolic stress. Biochim Biophy Acta. 2012;1819:1088-1097.

24. Kang Y, Fielden LF, Stojanovski D. Mitochondrial protein transport in health and disease. Semin Cell Dev Biol. 2018;76:142-153.

25. Austin S, St Pierre J. PGC1 $\alpha$ and mitochondrial metabolism: emerging concepts and relevance in ageing and neurodegenerative disorders. J Cell Sci. 2012;125:4963-4971.

26. Scarpulla RC. Metabolic control of mitochondrial biogenesis through the PGC-1 family regulatory network. Biochim Biophys Acta. 2011; 1813:1269-1278. 
27. Campello S, Strappazzon F, Cecconi F. Mitochondrial dismissal in mammals, from protein degradation to mitophagy. Biochim Biophys Acta. 2014;1837:451-460.

28. Wen-Xing Ding, Xiao-Ming Y. Mitophagy: mechanisms, pathophysiological roles, and analysis. Biol Chem. 2012;393:547-564.

29. Dolman NJ, Chambers KM, Mandavilli B, Batchelor RH, Janes MS. Tools and techniques to measure mitophagy using fluorescence microscopy. Autophagy. 2013:9:1653-1662.

30. Hamacher-Brady A, Brady NR. Mitophagy programs: mechanisms and physiological implications of mitochondrial targeting by autophagy. Cell Mol Life Sci. 2016;73:775-795.

31. Correia SC, Perry G, Moreira PI. Mitochondrial traffic jams in Alzheimer's disease: pinpointing the roadblocks. Biochim Biophys Acta. 2016; 1862:1909-1917.

32. Swomley AM, Förster S, Keeney JT, Triplett J, Zhang Z Sultana R et al. Abeta, oxidative stress in Alzheimer disease: evidence based on proteomics studies. Biochim. Biophys Acta. 2014;1842:1248-1257.

33. Smith MA. Alzheimer disease. Int Rev Neurobiol. 1998;42:1-54

34. Wang X, Su B, Lee HG, Li X, Perry G, Smith MA, et al. Impaired balance of mitochondrial fission and fusion in Alzheimer's disease. J Neurosci. 2009;29:9090-9103.

35. Manczak M, Reddy PH. Abnormal interaction between the mitochondria fission protein Drp1 and hyperphosphorylated tau in Alzheimer's disease neurons: implications for mitochondrial dysfunction and neuronal damage. Hum Mol Genet. 2012;21:2538-2547.

36. Baek SH, Park SJ, Jeong JI, Kim SH, Han J, Kyung JW, et al. Inhibition of Drp1 ameliorates synaptic depression, $A \beta$ deposition, and cognitive impairment in an Alzheimer's disease model. J Neurosci. 2017;37:5099-5110.

37. Kandimalla R, Manczak M, Yin X, Wang R, Reddy PH. Hippocampal phosphorylated tau induced cognitive decline, dendritic spine loss and mitochondrial abnormalities in a mouse model of Alzheimer's disease. Hum Mol Genet. 2018:27:30-40.

38. Sheng B, Wang X, Su B, Lee HG, Casadesus G, Perry G, et al. Impaired mitochondrial biogenesis contributes to mitochondrial dysfunction in Alzheimer's disease. J Neurochem. J Neurochem. 2012;120:419-429.

39. Hu Y, Li XC, Wang ZH, Luo Y, Zhang X, Liu XP, et al. Tau accumulation impairs mitophagy via increasing mitochondrial membrane potential and reducing mitochondrial Parkin. Oncotarget. 2016;7:17356-17368.

40. Wang L, Guo L, Lu L, Sun H, Shao M, Beck SJ, et al. Synaptosomal mitochondrial dysfunction in 5xFAD mouse model of Alzheimer's disease. PLoS One. 2016;11:e0150441.

41. Yan MH, Wang X, Zhu X. Mitochondrial defects and oxidative stress in Alzheimer disease and Parkinson disease. Free Radic Biol Med. 2013:62:90-101.

42. Sepúlveda-Falla D, Barrera-Ocampo A, Hagel C, Korwitz A Vinueza-Veloz MF, Zhou K, et al. Familial Alzheimer's disease-associated presenilin-1 alters cerebellar activity and calcium homeostasis. J Clin Invest. 2014;124:1552-1567.

43. Alexander GE. Biology of Parkinson's disease: pathogenesis and pathophysiology of a multisystem neurodegenerative disorder. Dialogues Clin Neurosci. 2004;6:259-280

44. Dauer W, Przedborski S. Parkinson's disease: mechanisms and models. Neuron. 2003:39:889-909.

45. Liang CL, Wang TT, Luby-Phelps K, German DC. Mitochondria mass is low in mouse substantianigra dopamine neurons: implications for Parkinson's disease. Exp Neurol. 2007;203:370-380.

46. Zhao F, Wang W, Wang C, Siedlak SL, Fujioka H, Tang B, et al. Mfn2 protects dopaminergic neurons exposed to paraquat both in vitro and in vivo: implications for idiopathic Parkinson's disease. Biochim Biophys Acta Mol Basis Dis. 2017:1863:1359-1370.

47. Santos D, Esteves AR, Silva DF, Januário C, Cardoso SM. The impact of mitochondrial fusion and fission modulation in sporadic Parkinson's disease. Mol Neurobiol. 2015;52:573-586.

48. Onyango IG, Khan SM, Bennett JP. Mitochondria in the pathophysiology of Alzheimer's and Parkinson's diseases. Front Biosci (Landmark Ed). 2017;22:854-872.

49. Stevens DA, Lee $Y$, Kang HC, Lee BD, Lee YI, Bower A, et al. Parkin loss leads to PARIS-dependent declines in mitochondrial mass and respiration. Proc Natl Acad Sci U S A. 2015;112:11696-11701.

50. Terman A, Kurz T, Navratil M, Arriaga EA, Brunk UT. Mitochondria turnover and aging of long-lived postmitotic cells: the mitochondrial-lysosomal axis theory of aging. Antioxid Redox Signal. 2010;12:503-535.
51. Nixon RA. The role of autophagy in neurodegenerative disease. Nat Med. 2013;19:983-997.

52. Schapira AH, Gegg M. Mitochondrial contribution to Parkinson's disease pathogenesis. Parkinsons Dis. 2011;2011:159160.

53. Ivankovic D, Chau KY, Schapira AH, Gegg ME. Mitochondrial and biogenesis are activated following PINK1/parkin-mediated mitophagy. J Neurochem. 2016;136:388-402.

54. Colpo GD, Stimming EF, Rocha NP, Teixeira AL. Promises and pitfalls of immune-based strategies for Huntington's disease. Neural Regen Res. 2017; $12: 1422-1425$

55. Montoya A, Price BH, Menear M, Lepage M. Brain imaging and cognitive dysfunctions in Huntington's disease. J Psychiatry Neurosci. 2006; 31:21-29.

56. McColgan P, Tabrizi SJ. Huntington's disease: a clinical review. Eur J Neurol. 2018;25:24-34

57. Waldvogel HJ, Kim EH, Thu DC, Tippett LJ, Faull RL. New perspectives on the neuropathology in huntington's disease in the human brain and its relation to symptom variation. J Huntingtons Dis. 2012;1:143-153.

58. Vonsattel JP, Myers RH, Stevens TJ, Ferrante RJ, Bird ED, Richardson EP. Neuropathological classification of Huntington's disease. J Neuropathol Exp Neurol. 1985;44:559-577.

59. Langbehn DR, Hayden MR, Paulsen JS, PREDICT-HD Investigators of the Huntington Study Group. CAG-repeat length and the age of onset in Huntington disease (HD): a review and validation study of statistical approaches. Am J Med Genet B Neuropsychiatr Genet. 2010; 153:397-408

60. Browne SE, Beal MF. The energetics of Huntington's disease. Neurochem Res. 2004;29:531-546.

61. Beal MF. Mitochondria take center stage in aging and neurodegeneration. Ann Neurol. 2005;58:495-505.

62. Kim J, Moody JP, Edgerly CK, Bordiuk OL, Cormier K, Smith K, et al. Mitochondrial loss, dysfunction and altered dynamics in Huntington's disease. Hum Mol Genet. 2010;19:3919-3935.

63. Shirendeb UP, Calkins MJ, Manczak M, Anekonda V, DufourB, McBride JL, et al. Mutant huntingtin's interaction with mitochondrial protein Drp1 impairs mitochondrial biogenesis and causes defective axonal transport and synaptic degeneration in Huntington's disease. Hum Mol Genet. 201;21:406-420.

64. Cui L, Jeong H, Borovecki F, Parkhurst CN, Tanese N, Krainc D. Transcriptional repression of PGC-1alpha by mutant hunting in leads to mitochondrial dysfunction and neurodegeneration. Cell. 2006;127:59-69.

65. Weydt P, Pineda VV, Torrence AE, Libby RT, Satterfield TF, Lazarowski ER, et al. Thermoregulatory and metabolic defects in Huntington's disease transgenic mice implicate PGC-1alpha in Huntington's disease neurodegeneration. Cell Metab. 2006;4:349-362.

66. Chaturvedi RK, Calingasan NY, Yang L, Hennessey T, Johri A, Beal MF. Impairment of PGC-1alpha expression, neuropathology and hepatic steatosis in a transgenic mouse model of Huntington's disease following chronic energy deprivation. Hum Mol Genet. 2010;19:3190-3205.

67. Pickrell AM, Youle RJ. The roles of PINK1, Parkin, and mitochondrial fidelity in Parkinson's disease. Neuron. 2015;85:257-273.

68. Ashrafi G, Schwarz TL. PINK1- and PARK2-mediated local mitophagy in distal neuronal axons. Autophagy. 2015;11:187-189.

69. Wang X, Winter D, Ashrafi G, Schlehe J, Wong YL, Selkoe D, et al. PINK1 and Parkin target Miro for phosphorylation and degradation to arrest mitochondrial motility. Cell. 2011:147:893-906.

70. Khalil B, El-Fissi N, Aouane A, Cabirol-Pol MJ, Rival T, Liévens JC. PINK1-induced mitophagy promotes neuroprotection in Huntington's disease. Cell Death Dis. 2015;22;6:e1617

71. Rui YN, Xu Z, Patel B, Chen Z, Chen D, Tito A, et al. Huntingtin functions as a scaffold for selective macroautophagy. Nat Cell Biol. 2015;17:262-275.

72. Chang DT, Rintoul GL, Pandipati S, Reynolds IJ. Mutant huntingtin aggregates impair mitochondrial movement and trafficking in cortical neurons. Neurobiol Dis. 2006;22:388-400.

73. Orr AL, Li S, Wang CE, Li H, Wang J, Rong J, et al. N-terminal mutant huntingtin associates with mitochondria and impairs mitochondrial trafficking. J Neurosci. 2008;28:2783-2792.

74. Oliveira JM. Nature and cause of mitochondrial dysfunction in Huntington's disease: focusing on huntingtin and the striatum. J Neurochem. 2010;114:1-12. 